\title{
Structure-activity associations in novel farrerol derivatives with vasorelaxant properties
}

\author{
XIAOMIN HOU ${ }^{1-3^{*}}$, XIAOJIANG QIN ${ }^{1,2,4^{*}}$ and QINGSHAN $\mathrm{LI}^{2,4}$ \\ ${ }^{1}$ School of Public Health; ${ }^{2}$ School of Pharmaceutical Science; \\ ${ }^{3}$ Department of Pharmacology, Shanxi Medical University, Taiyuan, Shanxi 030001; \\ ${ }^{4}$ School of Chinese Materia Medica, Shanxi University of Chinese Medicine, Jinzhong, Shanxi 030619, P.R. China
}

Received January 22, 2018; Accepted August 13, 2018

DOI: $10.3892 / \mathrm{mmr} .2018 .9439$

\begin{abstract}
To detect the structure-activity associations of farrerol derivatives, the relaxation activity of farrerol derivatives was observed in isolated aortic rings pre-contracted using phenylephrine in Sprague-Dawley rats. All compounds tested in the present study produced a relaxation effect, which was significantly affected by the molecular structure. Using a collagen gel contraction assay, the present study further evaluated the inhibitiory effect of farrerol derivatives in a decreased collagen gel area, induced by Angiotensin II. The results indicated that farrerol derivatives could inhibit collagen contraction, and that the inhibitory effect was associated with the molecular structure of the compounds. Furthermore, the inhibitory strength of the different compounds was consistent with the results of vascular tension detection. The activity of the farrerol derivatives was closely associated with the molecular structure. The analysis indicated that an electron-withdrawing substituent in the ortho position of the phenyl group (ring B) was crucial in order to observe improved vasorelaxation activity, whereas a hydroxyl or methoxy group was unfavorable. A para electron-donating group was oberved to increase compound activity. In addition, when the B ring was heterocycle rather than a phenyl ring, the vasorelaxation ability was weakened.
\end{abstract}

\section{Introduction}

Flavanone, an important natural compound, has great potential to reduce the risk of mortality in patients with cardiovascular diseases (1-3). Flavanone is widely distributed in plants, fruits

Correspondence to: Professor Qingshan Li, School of Pharmaceutical Science, Shanxi Medical University, 56 Xinjiannanlu, Taiyuan, Shanxi 030001, P.R. China

E-mail: sxlqs2012@163.com

*Contributed equally

Key words: farrerol derivatives, structure-activity associations, aorta, vasorelaxation, collagen contraction and vegetables, and consists of a large group of naturally existing polyphenolic compounds (4). Farrerol, a major bioactive component in the traditional Chinese herb 'Man-shan-hong' derived from the dried leaves of Rhododendron dauricum L., has numerous biological effects, including antithrombotic, anti-inflammatory, antioxidant, antiangiogenesis, vasorelaxant and antihypertensive effects (5-8). There has been great interest in the cardiovascular effects of flavonoids in humans. It has previously been reported that the greatest levels of vasorelaxant and antioxidant activity were detected in flavonols and flavones $(9,10)$. Previous research revealed that farrerol derivatives exhibited significant anti-atherosclerosis activity in rat vascular smooth muscle cells (VSMCs) and also exhibited strong cytoprotective activity against hydrogen peroxide-induced injury in human umbilical vein endothelial cells. Notably, they had preliminary structure-activity associations, indicating that farrerol derivatives could be used to treat and/or prevent cardiovascular disease $(11,12)$.

Farrerol has been demonstrated to induce a relaxing effect in the rat aorta (13); however, there is a lack of research regarding the structure-activity associations of farrerol derivatives with vasorelaxant properties. The present study aimed to use farrerol as the lead compound, and modify the structure to identify more potent vasorelaxant agents. In the present study, the vasorelaxant actions of farrerol derivatives in isolated rat thoracic aorta rings were examined, and the inhibitory effects of farrerol derivatives in rat aortic VSMCs contraction were investigated.

As we know, a greater understanding of the structural characteristics that optimize vasorelaxant activity is of critical importance to develop flavanone derivatives as potential therapeutic agents. Thus, the present study evaluated the structure-activity associations of farrerol derivatives in order to obtain highly active vasodilator compounds. The results may be useful for the treatment and/or prevention of vascular disease resulting from abnormal vascular contractility.

\section{Materials and methods}

Animals. A total of 120 Male Sprague-Dawley rats aged 12 weeks (weight, $210 \pm 20 \mathrm{~g}$ ) were obtained from the Animal Center of Shanxi Medical University (Taiyuan, China), with free access to food and water. Rats were housed five per 
cage. All of the rats were maintained at $22 \pm 3^{\circ} \mathrm{C}$ and $45 \pm 15 \%$ humidity with a normal 12-h light/dark cycle. All protocols were approved by the Animal Care and Use Committee of the Shanxi Medical University (Taiyuan, China), and all animal procedures were performed in accordance with the Ethical Guidelines for Animal Research in Shanxi Medical University.

Reagents. Phenylephrine (PE; purity $\geq 98 \%$ ), Angiotensin II (Ang II; purity $\geq 98 \%$ ) and Quercetin (purity $\geq 98 \%$ ) were purchased from Sigma-Aldrich (Merck KGaA, Darmstadt, Germany). Potassium chloride $(\mathrm{KCl})$, sodium chloride $(\mathrm{NaCl})$, magnesium chloride $\left(\mathrm{MgCl}_{2}\right)$, glucose, calcium chloride $\left(\mathrm{CaCl}_{2}\right)$ and the other reagents were of analytical purity and were purchased from Sangon Biotech Co., Ltd., (Shanghai, China). The purity of all of the farrerol derivatives was $>98 \%$, as determined by high performance liquid chromatography analysis; all of the compounds were synthesized according to the previously reported methods $(11,12)$ and the codes for all the compounds were presented in Table I. All of the farrerol derivatives used in these experiments were racemates. The basic structures of flavanone is illustrated in Fig. 1. Quercetin was used as a positive control drug. All of the farrerol derivatives and quercetin were dissolved in dimethyl sulfoxide (DMSO) and then diluted in distilled water in order to yield a final concentration of DMSO $<0.5 \%(\mathrm{v} / \mathrm{v})$.

Measurements of aortic ring tension. Male Sprague-Dawley rats were sacrificed by cervical dislocation in the presence of anesthesia with $5 \%$ chloral hydrate $(200 \mathrm{mg} / \mathrm{kg})$, then the blood was drained. The chests were opened, and the aortas were isolated then immediately transferred into $4^{\circ} \mathrm{C}$ Krebs-bicarbonate solution $(\mathrm{pH}=7.4)$, composed of $\mathrm{NaCl}$ $118.0 \mathrm{mM}, \mathrm{KCl} 4.7 \mathrm{mM}, \mathrm{KH}_{2} \mathrm{PO}_{4} 1.2 \mathrm{mM}, \mathrm{NaHCO}_{3} 25.0 \mathrm{mM}$, $\mathrm{CaCl}_{2} 2.5 \mathrm{mM}, \mathrm{MgCl}_{2}, 1.18 \mathrm{mM}$ and D-glucose $11.0 \mathrm{mM}$. The aortas were cleaned of adherent connective tissues, then were cut into $4 \mathrm{~mm}$ length rings.

Isolated rat aortic rings were suspended on two stainless steel hooks in baths containing $5 \mathrm{ml}$ of the Krebs-bicarbonate solution (maintained at $37^{\circ} \mathrm{C}$ and supplemented with $100 \% \mathrm{O}_{2}$ ). The upper hook was connected to a force transducer and changes in isometric force were recorded using Chart 5.4 (PowerLab; ADInstruments Inc., Colorado Springs, CO, USA). According to a previous study, passive tension was adjusted to $2 \mathrm{~g}$ and all subsequent measurements representing the force were generated above this baseline (14). Integrity of the endothelium was assumed when $10^{-6} \mathrm{M}$ acetylcholine induced $>70 \%$ relaxation of the aortic rings precontracted with $60 \mathrm{mM} \mathrm{KCl}$. A $2 \mathrm{~h}$ equilibration period was applied prior to any experimental relaxation effects of different farrerol derivatives to the aortic rings precontracted with $1 \mu \mathrm{M} \mathrm{PE}$ (15). When the contraction induced by $1 \mu \mathrm{M}$ PE was sustained, one of the drugs (different farrerol derivatives, positive control quercetin or vehicle DMSO) was added cumulatively to the chamber (13). Drugs at each concentration were added for $\sim 10 \mathrm{~min}$ at $37^{\circ} \mathrm{C}$. The end concentrations of farrerol derivatives or quercetin in the chamber were increased stepwise $(1,3,10,30$ and $100 \mu \mathrm{M})$. The relaxation response to a concentration of the farrerol derivatives was allowed to develop to a relatively stable plateau ( $\sim 10 \mathrm{~min})$, then the next concentration of farrerol derivative was added. The vasorelaxant activity of each farrerol derivative was detected in 6 aortic rings from 6 different rats (1 aortic ring/rat).

Cell culture andidentification . Rats were anesthetized by intraperitoneal administration of $5 \%$ chloral hydrate $(0.7 \mathrm{ml} / 100 \mathrm{~g})$, sacrificed by cervical dislocation, and then disinfected with $75 \%$ ethanol. The thoracic aorta was immediately removed under aseptic conditions, and was then rinsed thrice with PBS $\left(4^{\circ} \mathrm{C}\right)$ for $5 \mathrm{~min}$ each. The thoracic aorta was opened longitudinally. Rat aortic VSMCs were obtained from the medial layer as previously described (16). Briefly, the endothelium was gently scrapped off with a scalpel, then the vascular adventitia was carefully stripped with ophthalmic tweezers. The medial layer of the thoracic aorta was then cut into small pieces $\left(1 \mathrm{~mm}^{2}\right)$, which were plated into a tissue culture flask and were cultured in Dulbecco's modified Eagle's medium (DMEM) /F12 nutrient solution (Sigma Aldrich; Merck KGaA) supplemented with $20 \%$ fetal bovine serum (Sigma-Aldrich; Merck KGaA), L-glutamine (Sigma-Aldrich; Merck KGaA), streptomycin (100 U/ml; Sigma-Aldrich; Merck KGaA) and penicillin (100 U/ml, Sigma-Aldrich; Merck KGaA). The rat aortic VSMCs were verified through immunohistochemical staining of $\alpha$-actin. The VSMCs were cultured until they reached $80 \%$ confluency and were trypsinized $(0.05 \%)$ every 3 to 5 days. Cells between passages $4-8$ were used for subsequent experiments.

Cell contraction assay. Collagen gels were prepared as described previously $(17,18)$, then added to 24 -well culture dishes. Rat aortic VSMCs $\left(1 \times 10^{5}\right.$ cells per well) were seeded into wells containing $100 \mu \mathrm{l}$ neutralization solution and $400 \mu \mathrm{l}$ collagen, with a final collagen concentration of $1.5 \mathrm{mg} / \mathrm{ml}$. The VSMCs were then allowed to embed into the collagen gel. The VSMCs were cultured at $37^{\circ} \mathrm{C}$ for $24 \mathrm{~h}$, and were then used for the following treatments. Firstly, the original area of a collagen gel was measured. Ang II (10 ng/l) was added to cells at $37^{\circ} \mathrm{C}$ for $1 \mathrm{~h}$, then farrerol derivatives, quercetin or DMEM were added to cells at $37^{\circ} \mathrm{C}$ for $4 \mathrm{~h}$, successively. The collagen gel area was then measured. Gel images were captured using a camera following the administration of derivatives, and the areas $\left(\mathrm{cm}^{2}\right)$ of the gels were measured using ImageJ software version 1.50 (National Institutes of Health, Bethesda, MD, USA). Enhanced contractions were characterized by a decreased gel area, and reduced contractions were characterized by increased gel area. Changes to the surface area of collagen gels were expressed as a percentage relative to the untreated control. Three different fields were analyzed for each experiment that was performed.

Statistical analysis. All data are presented as mean \pm standard deviation. The $\mathrm{RC}_{50}$ was defined as the concentration of flavanone derivatives that induced a $50 \%$ reduction in the maximum relaxation from the contraction elicited by $1 \mu \mathrm{M}$ PE and was calculated from the concentration-response curve resulting from a nonlinear regression (curve fit) performed using GraphPad Prism 7.0 software (GraphPad Software, Inc., La Jolla, CA, USA). Statistical analysis was performed using the Student's paired t-test and one-way repeated measures analysis of variance followed by Bonferroni's post hoc test. $\mathrm{P}<0.05$ was considered to indicate a statistically significant difference. 
Table I. Vasorelaxant effects of all of the farrerol derivatives on phenylephrine-induced contractions in rat aortic rings.

\begin{tabular}{|c|c|c|c|c|c|c|c|}
\hline Compound & Structure & $\begin{array}{c}\text { Maximal } \\
\text { relaxation }(\%)\end{array}$ & $\mathrm{RC}_{50}(\mu \mathrm{M})$ & Compound & Structure & $\begin{array}{c}\text { Maximal } \\
\text { relaxation }(\%)\end{array}$ & $\mathrm{RC}_{50}(\mu \mathrm{M})$ \\
\hline a1 & & $65.13 \pm 2.12$ & 10.39 & $\mathrm{c} 1$ & & $98.44 \pm 5.41$ & 12.85 \\
\hline $\mathrm{a} 2$ & & $41.42 \pm 3.87$ & 69.47 & $\mathrm{c} 2$ & & $60.72 \pm 3.93$ & 22.45 \\
\hline a3 & & $48.78 \pm 4.45$ & 78.27 & d1 & & $96.38 \pm 3.65$ & 15.12 \\
\hline $\mathrm{a} 4$ & & $59.0 \pm 3.63$ & 15.64 & $\mathrm{~d} 2$ & & $68.45 \pm 4.09$ & 30.86 \\
\hline b1 & & $98.34 \pm 5.01$ & 14.68 & e1 & & $58.37 \pm 3.82$ & 74.38 \\
\hline b2 & & $47.44 \pm 3.76$ & 27.07 & $\mathrm{e} 2$ & & $88.92 \pm 4.16$ & 18.69 \\
\hline b3 & & $61.96 \pm 2.19$ & 20.97 & e3 & & $65.43 \pm 3.95$ & 52.34 \\
\hline
\end{tabular}

The RC50, concentration of flavanone derivatives that induced a $50 \%$ reduction in the maximum contraction elicited by $1 \mu \mathrm{M}$ phenylephrine.

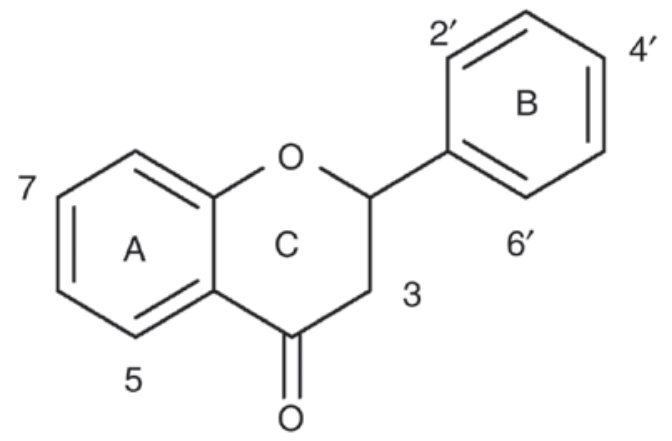

Figure 1. Structures of flavanone. The three rings contained in the flavanone structure were indicated by (A, B and C).

\section{Results}

Vasorelaxant activity of all compounds on the aorta pre-contracted by PE. Treatment with $1 \mu \mathrm{M}$ PE induced steady contractions in endothelium-intact rat aortic rings, and all of the farrerol derivatives examined at concentrations ranging from 1-100 $\mu \mathrm{M}$, produced concentration-dependent relaxation (Fig. 2), which could be reversed if the farrerol derivative was replaced with Krebs solution. The relative order of diastolic activity of the different farrerol derivatives examined in the present study is listed in Table I. In these experiments, the vasodilator amplitude of quercetin (positive control drug) in the isolated rat aortic rings was $55.74 \pm 4.19 \%$, which was less than that observed with farrerol $(65.13 \pm 2.12 \%)$. In addition, only three farrerol derivatives (a2, a3 and b2) had a weaker relaxation effect when compared with that observed following quercetin application to isolated rat aortas.

Results of cell contraction analysis. Collagen gels embedded with rat aortic VSMCs were prepared using primary cultured cells with very stable characteristics of the contractile phenotype. Collagen gel contraction experiments revealed a significant effect on gel surface area (enhanced contraction was characterized by decreased gel area and reduced contraction was characterized by increased gel area). The surface area of the collagen gels was significantly decreased upon the addition of Ang II (10 ng/l), indicating increased contractility. Collagen gel contractions were attenuated when incubated with farrerol derivatives in a dose-dependent manner and were relatively unaffected by DMEM (Fig. 3). The results of the cell contraction assay were consistent with of those of the aortic ring tension measurements. The inhibitory percentage of quercetin on the initial collagen area was $37.25 \pm 4.76 \%$, which was greater than that of farrerol $(20.48 \pm 3.52 \%)$. It was also revealed that only three farrerol derivatives (a2, a3 and b2) had greater inhibitory percentages than that of quercetin in regard to the initial collagen area.

\section{Discussion}

Farrerol, isolated from Rhododendron dauricum L., is a traditional Chinese medicine that has been reported to have beneficial effects for multiple conditions, including bronchitis and asthma (13). Our preliminary experiment determined the cytotoxicity of all of the farrerol derivatives in rat aortic VSMCs using MTT assays, the results revealed that there was no 
A

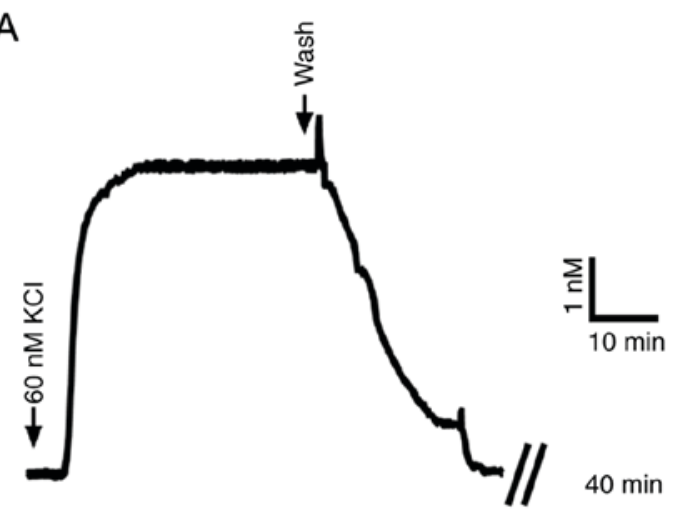

B
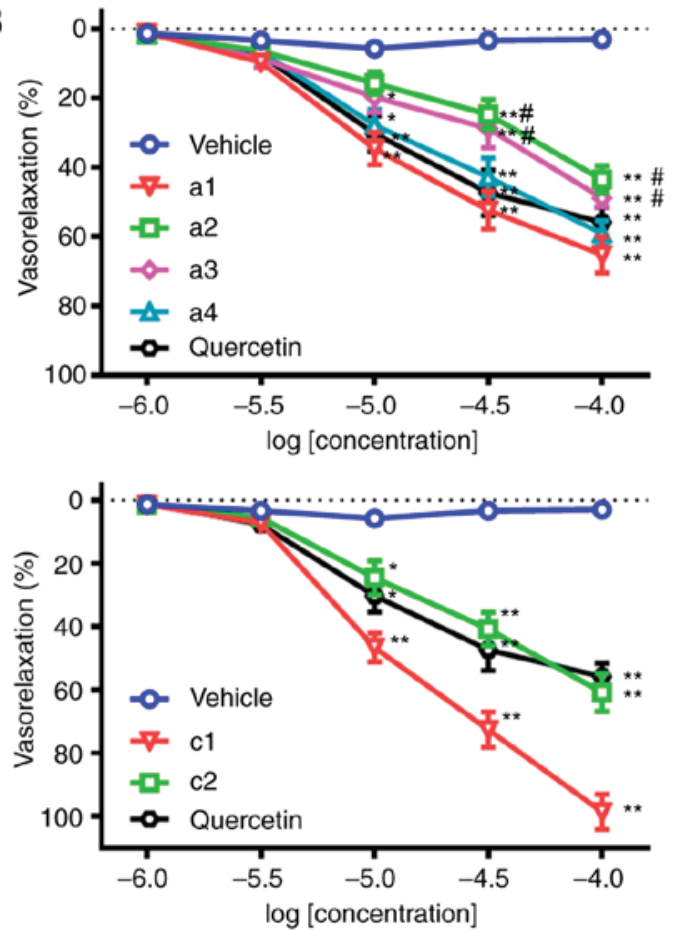

Farrerol derivatives $(\mu \mathrm{M})$
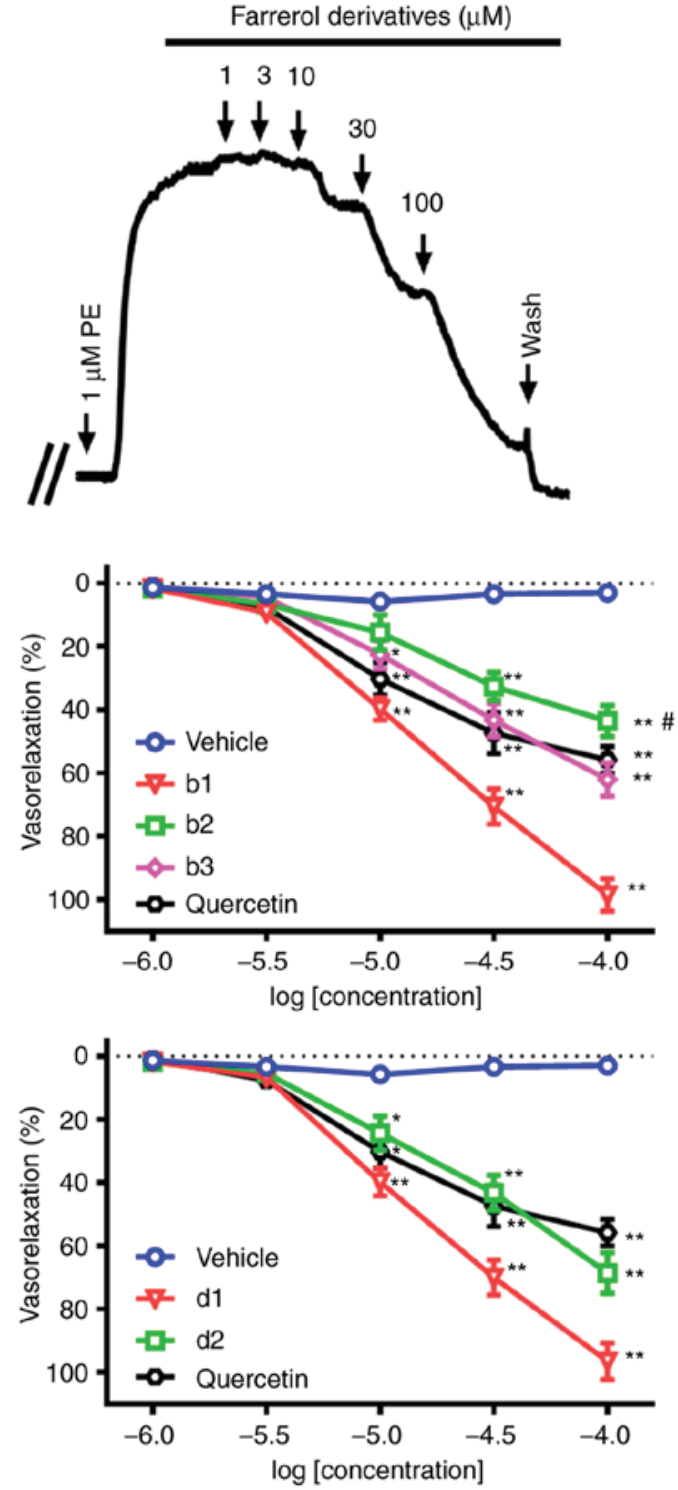

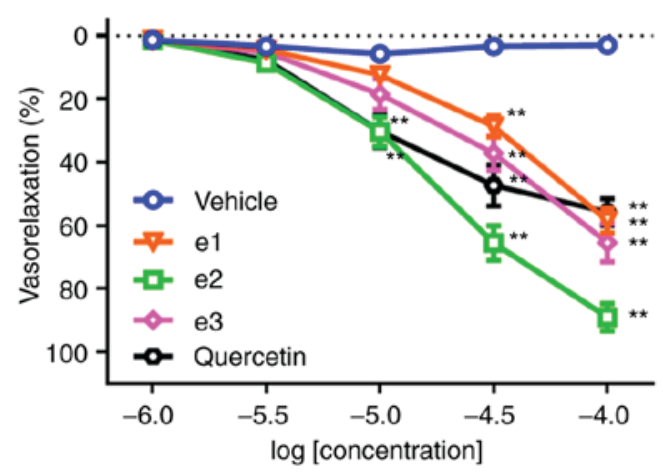

Figure 2. Concentration-response curves for the relaxation induced by different farrerol derivatives in isolated rat aortic rings pre-contracted with PE. (A) Original tension recording of the vasorelaxation induced by cumulative addition of farrerol derivatives on rat aortic rings pre-contracted with $1 \mu \mathrm{M}$ PE. When the precontraction was sustained, different concentrations $(1,3,10,30$ and $100 \mu \mathrm{M})$ of farrerol derivatives, positive control quercetin or vehicle were added to achieve the appropriate concentrations. (B) Pooled data (mean \pm standard deviation; $n=6$ ). Vasorelaxations were expressed as percentages of the pre-contraction induced by $1 \mu \mathrm{M}$ PE. The farrerol derivatives were presented in Table I (a1-a4, b1-b3, c1-c2, d1-d2, e1-e3). "P<0.05 and ${ }^{* * *} \mathrm{P}<0.01$ vs. vehicle group; ${ }^{\#} \mathrm{P}<0.05$ vs. quercetin group. $\mathrm{PE}$, phenylephrine.

cytotoxicity up to $60 \mu \mathrm{M}$ (data not shown). Our previous study demonstrated that farrerol could induce relaxation in rat aortic rings precontracted by PE in a dose-dependent manner (13). Owing to the structural similarity of farrerol derivatives to that of farrerol, farrerol was also used to explore the possible mechanisms involved as the lead compound. It was hypothesized that the relaxation effect of farrerol derivatives may be associated with the molecular structure, which was confirmed by two different experimental methods (the rat aortic tension test and collagen gel contraction assay) in the present study. 
A

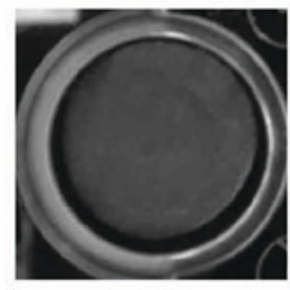

Control

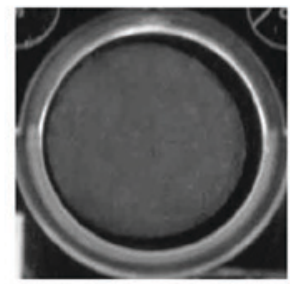

Control

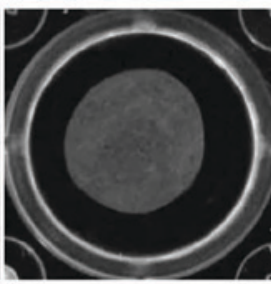

Ang II

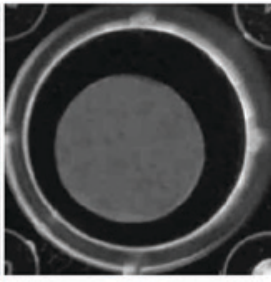

Ang II
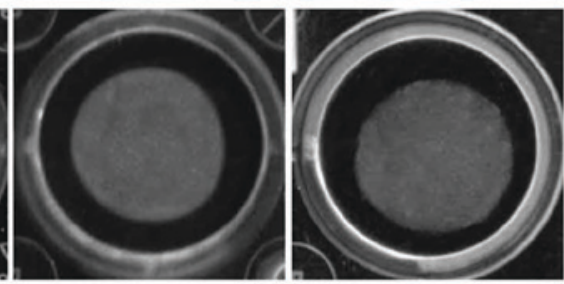

DMEM

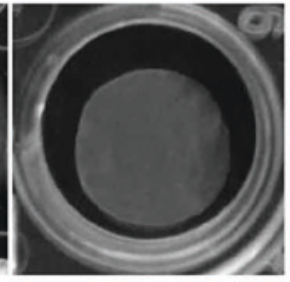

$3 \mu \mathrm{M}$

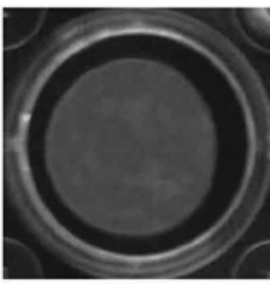

$10 \mu \mathrm{M}$
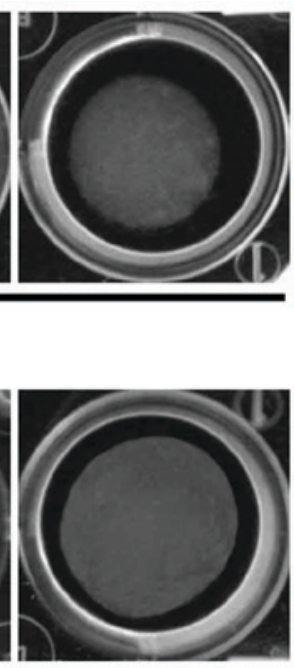

$30 \mu \mathrm{M}$

Farrerol derivatives
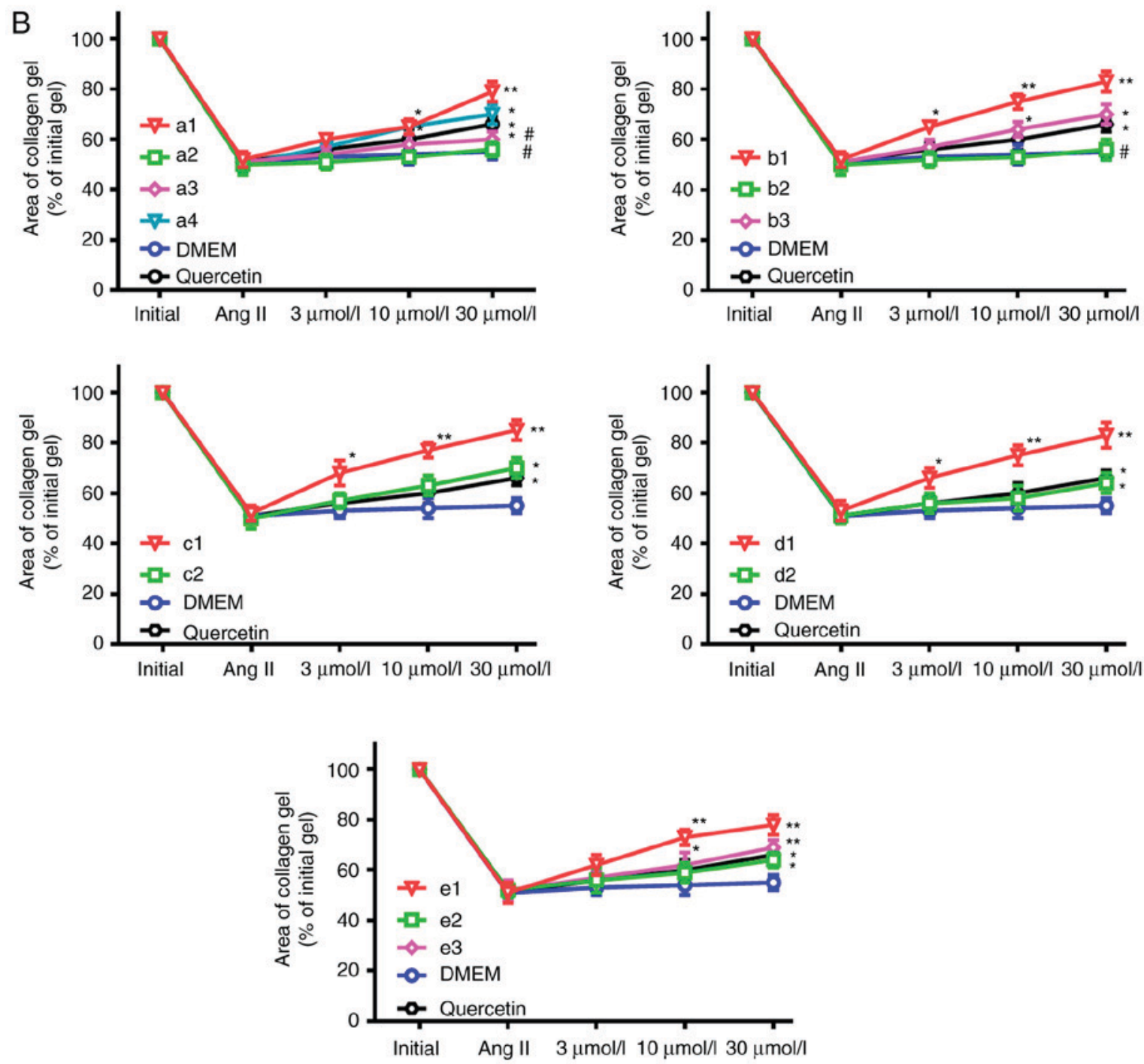

Figure 3. Effects of different farrerol derivatives on collagen gel contraction. (A) A representative photograph of collagen gel contraction. Ang II (10 ng/l) caused collagen gel/VSMCs contractions, which were attenuated by farrerol derivatives or positive control quercetin in a concentration-dependent manner $(3,10$ and $30 \mu \mathrm{M})$ and were relatively unaffected by DMEM (magnification, x100). (B) Comparisons of the collagen gel surface area following the application of different farrerol derivatives, positive control quercetin or DMEM. Changes in collagen gel surface area were expressed as the ratio of the experimental gel area to that of the untreated control. Three different fields were analyzed for each experiment that was performed $(\mathrm{n}=3)$. The farrerol derivatives were presented in Table I (a1-a4, b1-b3, c1-c2, d1-d2, e1-e3). " $\mathrm{P}<0.05$ and ${ }^{* *} \mathrm{P}<0.01$ vs. DMEM group; ${ }^{*} \mathrm{P}<0.05$ vs. quercetin group. Ang II, Angiotensin II; VSMCs, vascular smooth muscle cells; DMEM, Dulbecco's modified Eagle's medium. 
Our previous research confirmed that farrerol could relax precontracted rat aortic rings and inhibit contractions produced by a vasoconstrictor (13). Furthermore, it was demonstrated that farrerol could reduce $\left[\mathrm{Ca}^{2+}\right]_{\text {in }}$ in cultured VSMCs by laser scanning confocal microscopy and suppress $\mathrm{Ca}^{2+}$ influx via L-type voltage gated $\mathrm{Ca}^{2+}$ channel via the patch clamp technique (13). These results suggested that the antihypertensive effect of farrerol could be explained by the direct vasodilatory effect of farrerol on VSMCs. VSMCs serve an important role in maintaining blood pressure and facilitating the flow of nutrients in the body (19). PE could cause aortic contraction by $\mathrm{Ca}^{2+}$ influx through reactive oxygen cluster (ROC) and by the release of $\mathrm{Ca}^{2+}$ from the sarcoplasmic reticulum $(20,21)$. In our previous experiments, farrerol relaxed the precontraction in the rat aortic rings induced by $\mathrm{PE}$, implying that farrerol may decrease $\mathrm{Ca}^{2+}$ influx by blocking ROC and inhibiting $\mathrm{Ca}^{2+}$ release from the sarcoplasmic reticulum (13). In the present study, farrerol derivatives were evaluated for their vasodilatory effect on rat aortic rings precontracted by $\mathrm{PE}$. The results demonstrated that all of the examined farrerol derivatives exhibited a vasorelaxation effect in rat aortic rings; however, the vasorelaxation activity varied between the derivatives. Compounds $\mathrm{c} 1, \mathrm{~d} 1$ and $\mathrm{b} 1$ exhibited high vasorelaxation activity, the maximum relaxation percentages were $98.44 \pm 5.41,96.38 \pm 3.65$ and $98.34 \pm 5.01 \%$, respectively $\left(\mathrm{RC}_{50}=12.85,15.12\right.$ and $14.68 \mu \mathrm{M}$, respectively). The results indicated that compounds are more likely to have positive vasorelaxation activity if they have an electron-withdrawing substituent in the ortho position of the phenyl group (ring B). This is due to compounds $\mathrm{cl}$ and $\mathrm{d} 1$, which have an ortho nitro group on the $\mathrm{B}$ ring, having the strongest vasorelaxation activity; whereas, compounds $\mathrm{c} 2$ and $\mathrm{d} 2$, with a para electron withdrawing group, displayed weak vasorelaxation activity. Notably, when there was no substituent on the B ring (b1), the compound could also exert good vasodilatation activity.

In the present study, it was also revealed that compounds a1-a4 and e1-e3 exhibited a vasorelaxation effect on precontracted rat aortic rings. The results indicated that an ortho electron-withdrawing substituent is crucial for the vasorelaxation activity, whereas, a hydroxyl or methoxy group is unfavorable. A number of studies have reported that the extra hydroxyl group present in the flavanone at the $\mathrm{C} 3$ position is important for vascular activity as they have greater vasorelaxation effects. In addition, the pattern of substitution of hydroxyl groups on the A and B rings of compounds also influences activity $(22,23)$, particularly substitution on the $\mathrm{B}$ ring, as flavonols with a $\mathrm{C}_{3^{\prime}, 4^{\prime}} \mathrm{diOH}$ or $\mathrm{C}_{3^{\prime}, 4^{\prime}, 5^{\prime}}$ triOH orientation exhibited weak vasodilation activity (24). A para electron-withdrawing group or an electron-donating group could also decrease the anti-tumor activities of farrerol derivatives in vitro (11). As demonstrated in the present study, the results indicated that a para electron-donating group increased the vasorelaxation effect, as compounds a1 and e 2 exhibited high vasodilation activity. In addition, when the heterocycle is a B ring instead of a phenyl group, the vasorelaxation ability of compounds b2 and b3 were weaker than b1. The results indicated that a heterocycle was unfavorable for relaxation activity. Furthermore, the relaxation effect was greatly influenced by the molecular structure of the different farrerol derivatives.

Unimpaired VSMC function is essential for the maintenance of life. In the present study, using a collagen gel contraction assay, the inhibitory effect of farrerol derivatives in decreased collagen gel area induced by Ang II was examined. Ang II has been reported to be associated with cardiac growth and hypertensive disease $(25,26)$. The farrerol derivatives examined in the present study were able to inhibit collagen gel/VSMC contraction in a concentration-dependent manner, and the inhibitory strength of different farrerol derivatives was also consistent with the results of the myogenic experiments. Furthermore, the inhibitory activity of farrerol derivatives on collagen gel/VSMC contraction were enhanced by an ortho electron-withdrawing substituent or a para electron-donating group on the B ring, but were weakened by a para electron withdrawing group or a heterocycle on the B ring as opposed to a phenyl group.

Based on the structure-activity associations of farrerol derivatives established in the present study, it was hypothesized that an ortho electron-withdrawing substituent may be crucial for the vascular activity of the farrerol derivatives, whereas, a hydroxyl or methoxy group may be unfavorable. A para electron-donating group increased the activity of the examined compounds. Meanwhile, it was unfavorable for compound activity when the heterocycle was on the B ring, as opposed to a phenyl group. Further in vivo and in vitro tests of different farrerol derivatives are currently underway. These will provide important information for further structure modifications and the results of the present study will hopefully provide a basis to develop novel anti-cardiovascular drug candidates. The molecular structure of farrerol contains an S configuration; however, the $\mathrm{S}$ configuration and its determination have yet to be investigated. In the future research, we will confirm the enantiopurity of farrerol derivatives by typical polarimetric techniques in order to elucidate whether they interact with raceme or enantiomers. In the present study, the main focus was on the structure-vasodilatation activity associations of farrerol and its derivatives on the isolated aortic rings and rat aorta VSMCs. Our ongoing research will further explore the mechanism of farrerol derivatives relaxing the aorta in rats.

In conclusion, the type and position of the substituents on the $\mathrm{B}$ ring serve an important role in the relaxation activity of farrerol derivatives. An ortho electron-withdrawing substituent was crucial for the activity of the farrerol derivatives, whereas a hydroxyl or methoxy group was unfavorable. A para electron-donating group could increase the activity of the compounds. In the future, the group will continue to synthesize the corresponding derivatives with $-\mathrm{NH}_{2}$ or $-\mathrm{SO}_{3} \mathrm{H}$ in the ortho or para positions. In addition, the heterocyclic structure was unfavorable for compound activity when the heterocycle was on the $\mathrm{B}$ ring instead of a phenyl group. The effects of compounds with different hydroxyl and vinyl groups in the $\mathrm{C}$ ring should also be considered; thus, the group will continue to modify the $\mathrm{C}$ ring to acquire highly active compounds. The present study only investigated the structure-activity associations of a racemate mixture of farrerol derivatives on the rat aorta. In future experiments, enantiomers from farrerol derivatives will be separated and studied to elucidate their vasorelaxant activity in rat aortas.

The results of the present study have facilitated the identification of the structural characteristics that promote the vasorelaxation activity of farrerol derivatives, which may lead to the development of agents useful in the treatment of cardiovascular disease. 


\section{Acknowledgements}

Not applicable.

\section{Funding}

The present study was financially supported by the Youth Science and Technology Research Fund of Shanxi Province (grant nos. 201701D221247 and 201701D221259), the Science and Technology Innovation Project of Shanxi Higher School (grant nos. 2017146 and 2017147), the Youth Fund of Shanxi Medical University (grant nos. 02201604 and 02201613), the Startup Foundation for Doctors of Shanxi Medical University (grant nos. 03201510 and 03201521) and the Fund for Shanxi '1331 Project' Key Subjects Construction.

\section{Availability of data and materials}

All data generated or analyzed during this study are included in this published article.

\section{Authors' contributions}

$\mathrm{XH}$ and $\mathrm{XQ}$ performed the experiments and were the major contributors in writing the manuscript. QL conceived and designed the study, and was involved in drafting the manuscript and critically revising the manuscript for important intellectual content. All authors have read and approved the final manuscript.

\section{Ethics approval and consent to participate}

All animal experimental protocols were approved by the Animal Care and Use Committee of the Shanxi Medical University (Taiyuan, China), and were performed in accordance with the Ethical Guidelines for Animal Research in Shanxi Medical University.

\section{Patient consent for publication}

Not applicable.

\section{Competing interests}

The authors declare that they have no competing interests.

\section{References}

1. Da Pozzo E, Costa B, Cavallini C, Testai L, Martelli A, Calderone $\mathrm{V}$ and Martini $\mathrm{C}$ : The citrus flavanone naringenin protects myocardial cells against age-associated damage. Oxid Med Cell Longev 2017: 9536148, 2017.

2. Goetz ME, Judd SE, Hartman TJ, Mcclellan W, Anderson A and Vaccarino V: Flavanone intake is inversely associated with risk of incident ischemic stroke in the REasons for geographic and racial differences in stroke (REGARDS) study. J Nutr 146 2233-2243, 2016

3. Chin KY, Silva LS, Darby IA, Ng DCH and Woodman OL: Protection against reperfusion injury by 3 ',4'-dihydroxyflavonol in rat isolated hearts involves inhibition of phospholamban and JNK2. Int J Cardiol 254: 265-271, 2018.

4. Ajay M, Gilani AU and Mustafa MR: Effects of flavonoids on vascular smooth muscle of the isolated rat thoracic aorta. Life Sci 74: 603-612, 2003.
5. Dai F, Gao L, Zhao Y, Wang C and Xie S: Farrerol inhibited angiogenesis through Akt/mTOR, Erk and Jak2/Stat 3 signal pathway. Phytomedicine 23: 686-693, 2016.

6. Li JK, Ge R, Tang L and Li QS: Protective effects of farrerol against hydrogen-peroxide-induced apoptosis in human endothelium-derived EA.hy926 cells. Can J Physiol Pharmacol 91: 733-740, 2013.

7. Nijveldt RJ, van Nood E, van Hoorn DE, Boelens PG, van Norren K and van Leeuwen PA: Flavonoids: A review of propable mechanisms of action and potential application. Am J Clin Nutr 74: 418-425, 2001.

8. Qin X, Hou X, Zhang K and Li Q: Farrerol modulates aorta gene expression profile in spontaneously hypertensive rats. Planta Med 84: 296-303, 2018.

9. Qin X, Lu Y, Peng Z, Fan S and Yao Y: Systematic chemical analysis approach reveals superior antioxidant capacity via the synergistic effect of flavonoid compounds in red vegetative tissues. Front Chem 6: 9, 2018

10. Zeng X, Xi Y and Jiang W: Protective roles of flavonoids and flavonoid-rich plant extracts against urolithiasis: A review. Crit Rev Food Sci Nutr 12: 1-11, 2018.

11. Shi L, Feng XE, Cui JR, Fang LH, Du GH and Li QS: Synthesis and biological activity of flavanone derivatives. Bioorg Med Chem Lett 20: 5466-5468, 2010.

12. Shi L, Feng XE, Lin WH, Fang LH, Du GH and Li QS: Synthesis of new flavanone derivatives of farrerol and preliminary SAR studies on anti-VSMCs vegetation activity. Chem Res Chin Univ 27: 237-240, 2011

13. Qin X, Hou X,Zhang M, Liang T,Zhi J, Han L and Li Q: Relaxation of rat aorta by farrerol correlates with potency to reduce intracellular calcium of VSMCs. Int J Mol Sci 15: 6641-6656, 2014.

14. Wong ESW, Man RYK, Ng KFJ, Leung SWS and Vanhoutte PM: $\mathrm{L}$-arginine and arginase products potentiate dexmedetomidine-induced contractions in the rat aorta. Anesthesiology 128: 564-573, 2018

15. Niu LG, Zhang MS, Liu Y, Xue WX, Liu DB, Zhang J and Liang YQ: Vasorelaxant effect of taurine is diminished by tetraethylammonium in rat isolated arteries. Eur J Pharmacol 580: $169-174,2008$

16. Xu S, Fu J, Chen J, Xiao P, Lan T, Le K, Cheng F, He L, Shen X, Huang $\mathrm{H}$ and Liu P: Development of an optimized protocol for primary culture of smooth muscle cells from rat thoracic aortas. Cytotechnology 61: 65-72, 2009.

17. Zagai U, Sköld CM, Trulson A, Venge P and Lundahl J: The effect of eosinophils on collagen gel contraction and implications for tissue remodelling. Clin Exp Immunol 135: 427-433, 2004.

18. Kohyama T, Liu X, Wen FQ, Zhu YK, Wang H, Kim HJ, Takizawa H, Cieslinski LB, Barnette MS and Rennard SI: PDE4 inhibitors attenuate fibroblast chemotaxis and contraction of native collagen gels. Am J Respir Cell Mol Biol 26: 694-701, 2002.

19. Thorneloe KS and Nelson MT: Ion channels in smooth muscle: Regulators of intracellular calcium and contractility. Can J Physiol Pharmacol 83: 215-242, 2005.

20. Dellis O, Dedos SG, Tovey SC, Taufiq-Ur-Rahman, Dubel SJ and Taylor CW: Ca2+entry through plasma membrane IP3receptors. Science 313: 229-233, 2006.

21. Xia M, Qian L, Zhou X, Gao Q, Bruce IC and Xia Q: Endothelium-independent relaxation and contraction of rat aorta induced by ethyl acetate extract from leaves of Morus alba (L.). J Ethnopharmacol 120: 442-446, 2008.

22. Dugas AJ, Castañeda-AJ, Bonin GC, Price KL, Fischer NH and Winston GW: Evaluation of the total peroxyl radical-scavenging capacity of flavonoids: Structure-activity relationships. J Nat Prod 63: 327-331, 2000.

23. Masek A, Chrzescijanska E, Latos M and Zaborski M: Influence of hydroxyl substitution on flavanone antioxidants properties. Food Chem 215: 501-507, 2017.

24. Herrera MD, Zarzuelo A, Jiménez J, Marhuenda E and Duarte J: Effects of flavonoids on rat aortic smooth muscle contractility: Structure-activity relationships. Gen Pharmacol 27: 273-277, 1996.

25. Simo-Cheyou ER, Ju JT, Grygorczyk R and Srivastava AK: STIM-1 and ORAI-1 channel mediate angiotensin-II-induced expression of Egr-1 in vascular smooth muscle cells. J Cell Physiol 232: 3496-3509, 2017.

26. Caillon A, Mian MOR, Fraulob-Aquino JC,Huo KG, Barhoumi T, Ouerd S, Sinnaeve PR, Paradis P and Schiffrin EL: $\gamma \delta$ T cells mediate angiotensin II-induced hypertension and vascular injury. Circulation 135: 2155-2162, 2017. 